\title{
Percepción de la relación entre educación superior y trabajo en graduados de la Universidad Tecnológica de El Salvador
}

URI: http://hdl.handle.net/11298/285

Paola María Navarrete Ruth Maritza Osegueda ${ }^{2}$

paola.navarrete@utec.edu.sv

Recibido: 24/08/16 - Aceptado: 19/09/16

\section{Resumen}

En este artículo se profundiza en uno de los resultados obtenidos en la investigación institucional "Posicionamiento en el ámbito laboral y caracterización de graduados universitarios de la Universidad Tecnológica de El Salvador", que tuvo como objetivo desarrollar un perfil y caracterización del graduado de la Universidad Tecnológica de El Salvador (Utec). Este artículo se centra en las percepciones que tienen los graduados sobre la relación entre su formación universitaria y su desarrollo profesional, incluyendo su capacidad de obtener un empleo, así como la relación entre su actual empleo y su carrera. Entre los resultados se obtuvo que el 63,9\% de los graduados está ejerciendo su trabajo en áreas relacionadas con su formación académica. Esta percepción es más notoria en graduados del sexo masculino y en aquellos pertenecientes a zonas rurales. Los graduados también manifestaron que su formación académica ha sido de utilidad y ayuda en su empleo, permitiéndoles desempeñarse mejor, pero que no tiene relación directa con obtener un empleo o mejorarlo.

\section{Palabras clave}

Socialización profesional, Universidad Tecnológica de El Salvador - Educación - El Salvador, Graduados universitarios.

\section{Abstract}

This article delves into one of the results obtained in the institutional research entitled "Job positioning and characterization of graduates from Universidad Tecnológica de El Salvador (Utec). This article focuses on the perceptions graduates have in terms of the relationship between their academic training and their professional development, including their capacity to be employed, as well as the relation between their current job and their major. The results showed the following facts: $63,9 \%$ of graduates is currently working in fields related to their academic training. This perception becomes more obvious in males and in those who belong to the rural areas. Though the graduates also pointed out that their academic training has been useful and helpful in their job thus allowing them to perform better, this has no direct relation to being able to get a job or improving the opportunity for a better job.

\section{Keywords}

Professional Socialization, Universidad Tecnológica de El Salvador-Education-El Salvador, University graduates.

\footnotetext{
1 Antropóloga, investigadora de la Universidad Tecnológica de El Salvador. paola.navarrete@utec.edu.sv

2 Licenciada en Psicología, colaboradora en la investigación.
} 


\section{Introducción}

La actual tendencia en el mundo globalizado y en el mercado laboral presenta características que han permitido la adopción de estrategias similares a las que tradicionalmente se utilizan para lanzamientos y promoción de productos, pero dirigidas hacia profesionales, principalmente enfocadas hacia jóvenes y personas que ingresan por primera vez al mercado de trabajo, o en aquellos que cambian de sector o modalidad de trabajo (Rentería y Martínez, 2006b).

Estos nuevos escenarios, a los que se deben incorporar los egresados universitarios, demandan cada vez más una sólida formación profesional, adecuada a exigencias de mercados cada día más competitivos, requiriendo que el profesional involucre con inteligencia sus capacidades para insertarse al mercado laboral de manera competitiva. Las demandas en el mercado laboral ya no se enfocan hacia profesionales con sólidos conocimientos científicos y técnicos en una disciplina específica; se requiere además de una fuerte formación en competencias, consideradas relevantes para su desempeño profesional (Betnaces, 2007). Es por eso que se hace necesario que las instituciones de educación superior, que forman a profesionales que irán a desempeñarse al mercado laboral, conozcan y entiendan hacia qué mercados están capacitando a sus estudiantes y graduados; y comprender si esas competencias y capacidades son las adecuadas para competir y dar un aporte al mundo laboral.

Ahora el graduado debe logar posicionarse en el área en la cual ha estado formando sus competencias para aportar conocimientos, habilidades y actitudes a su entorno laboral. En el contexto del trabajo, los sujetos pueden apropiarse o desarrollar recursosque les permitan lograr un posicionamiento, siempre y cuando los demás sujetos inmersos en ese contexto reconozcan determinados aspectos, como el liderazgo, la productividad, la adecuada comunicación y una buena actitud (Hall, 1996).

Tradicionalmente el término posición se interpreta desde el punto de vista de la mercadotecnia, que se refiere al lugar y los atributos que el cliente percibe mentalmente de un producto. La diferencia entre posición y posicionamiento, en el caso del mercado laboral, implica no solo el lugar en el que se ubica el sujeto, sino además el reconocimiento que los otros hagan de dicha ubicación. El mercado laboral, como contexto, permite que las personas se encuentren y se reconozcan; este espacio de interrelaciones les permite construir su identidad, y cada sujeto toma una posición frente a dicho contexto (Rentería y Enríquez, 2006; citado en Carillo, Escobar, y Audelo, 2013).

\section{Posicionamiento de graduados universitarios}

Pero a escala nacional no existen instituciones, a veces ni siquiera dentro de las propias universidades, que velen y den seguimiento a sus graduados universitarios. Por lo general no se sabe cuántos de ellos están insertados en el mundo laboral, si trabajan en áreas relacionadas con sus estudios, ni de qué manera sus estudios ayudaron a colocarlos en un trabajo o si les permitió una mejor posición que en la que se encontraban. Un mayor entendimiento de la relación que existe, para los graduados, entre sus estudios con su experiencia en la búsqueda de un empleo, e incluso ya ejerciendo su profesión, permite entender qué otras competencias, habilidades 0 conocimientos necesita un profesional para ser competente y competitivo profesionalmente.

Han existido en El Salvador pocos informes e investigaciones por parte de universidades, u otros entes, que determinen el posicionamiento que tienen los graduados en el ámbito laboral salvadoreño. Generalmente se han realizado con el propósito de identificar en qué áreas han logrado posicionarse los egresados de sus instituciones. Estos estudios realizan un diagnóstico que, a su vez, permite crear mecanismos que ayuden a la incorporación de los egresados de sus universidades al mundo laboral, como además mejorar los procesos educativos desarrollados dentro de su institución (Ruiz y Green, 2003; Universidad Modular Abierta [UMA], 2012). Las transformaciones técnico-científicas han requerido nuevos modos de organización, tanto para los procesos de trabajo como para los de formación. Este escenario demanda acciones constantes de seguimiento y evaluación de los egresados, ya sea para la reorganización interna de los programas o para la proposición de nuevos (Programa ALFA América Latina, 2006).

Es posible que existan otros estudios que hablen de los graduados en otras universidades, pero también que esta información se quede a nivel institucional y no sea divulgada en la comunidad científica. Queda pendiente - y es una deuda por parte de las universidades salvadoreñas - realizar un estudio de seguimiento a escala nacional que pueda brindar a la población diagnósticos actualizados del posicionamiento de egresados universitarios, mostrando tendencias y necesidades. 


\section{Método}

\section{Participantes o sujetos de estudio}

El estudio tomó en cuenta a graduados de licenciatura pertenecientes a la Utec dentro del período 2011-2015, considerando a hombres y mujeres de las diferentes carreras que imparte.

\section{Universo y muestra}

Desde el año 2011 hasta el 2014 hay un registro de 6.724 graduados (Mined, 2015). Con este universo, se estimó una muestra total de 252 participantes para el estudio, con base en la fórmula para el cálculo de poblaciones finitas, la cual responde a un nivel de confianza estimado del $95 \%$ y un margen de error del $6 \%$, con distribución y selección al azar.

\section{Descripción de la población encuestada}

Entre los datos sociodemográficos de la población sondeada (ver Tabla 1. Características sociodemográficas de los participantes), 136 de los encuestados son del sexo masculino, representando el 54,5\%; mientras que 113 (45,5\%) corresponden al sexo femenino. Según el área de residencia, son pertenecientes al sector urbano el 91,5\% $(n=225)$; y para el área rural, el 8,5\% $(n=21)$. En la variable Estado civil, se tiene que el $42,8 \%(n=108)$ está casado; un $40,6 \%(n=102)$ es soltero; aquellos que manifestaron estar acompañados son un $11,7 \%(n=29)$; y divorciados, con $4,4 \%(n=11)$, entre las variables que caracterizan a la muestra encuestada.

\section{Diseño y tipo de estudio}

La metodología implementada fue cuantitativa, mediante un estudio descriptivo con diseño trasversal y exploratorio (Hernández, Fernández y Baptista, 2014).

\section{Instrumento utilizado para la recolección de datos}

Para esta investigación se utilizó una encuesta estructurada de tipo cerrado (Hernández, Fernández y Baptista, 2014), conformada por ítems de nivel nominal dicotómico, polinómico y de respuesta variable cerrada. Para la validación de los instrumentos se realizó un análisis de contenido, para mediar la congruencia de los ítems con el estudio e identificar las principales variables que predicen el posicionamiento laboral. Posteriormente se realizó un análisis factorial exploratorio sobre los ítems validados, y se obtuvo un nivel de congruencia interna del instrumento suficiente para garantizar la cohesión de los ítems y su consecuente predicción de la variable interna.

Tabla 1. Características sociodemográficas de los participantes

\begin{tabular}{lccc}
\hline & Variables & Recuento & Porcentaje \\
\hline Sexo: & Femenino & 113 & 45,5 \\
Estado civil: & Masculino & 136 & 54,5 \\
& Soltero & 102 & 40,6 \\
& Casado & 108 & 42,8 \\
& Acompañado & 29 & 11,7 \\
Zona: & Divorciado & 11 & 4,4 \\
& Viudo & 1 & 0,6 \\
Departamento & Urbano & 225 & 91,5 \\
& Rural & 21 & 8,5 \\
& Ahuachapán & 6 & 2,2 \\
& Cabañas & 3 & 1,1 \\
& Chalatenango & 1 & 0,6 \\
& Cuscatlán & 13 & 5,0 \\
& La Libertad & 46 & 18,4 \\
& La Paz & 3 & 1,1 \\
& San Salvador & 176 & 70,4 \\
& San Vicente & 1 & 0,6 \\
& Usulután & 1 & 0,6 \\
\hline
\end{tabular}




\section{Procedimiento para recolección de datos}

La encuesta se realizó de manera virtual. Esta técnica de recolección de información fue factible debido a que la muestra de graduados tiene acceso a internet y a correo electrónico, necesario para recibir la información de la encuesta; se les explicó vía correo en qué consistía la investigación, cómo desarrollar la encuesta y la importancia de su aporte.
Al comparar estos resultados con algunas variables demográficas podemos distinguir, si bien sin significancia, diferencias entre la población.

Los graduados del sexo masculino manifestaron tener una percepción de mayor relación entre su actual trabajo y su formación, en comparación con las mujeres. Teniendo la población masculina una media de 1.42, en comparación con 1.58 de las mujeres.

Tabla 2. Relación entre carrera y posicionamiento laboral

\begin{tabular}{lcccc}
\hline Frecuencia & Porcentaje & $\begin{array}{c}\text { Porcentaje } \\
\text { válido }\end{array}$ & $\begin{array}{c}\text { Porcentaje } \\
\text { acumulado }\end{array}$ \\
\hline Mucho & 161 & 63,9 & 66,1 & 66,1 \\
Algo & 55 & 21,7 & 22,4 & 88,5 \\
Poco & 18 & 7,2 & 7,5 & 96,0 \\
Nada & 10 & 3,9 & 4,0 & 100,0 \\
Total & 244 & 96,7 & 100,0 & \\
Perdido sistema & 8 & 3,3 & & \\
\hline
\end{tabular}

\section{Resultados}

\section{Coherencia entre la formación y el tipo de empleo}

Se preguntó a los graduados si el trabajo que están desempeñando tiene o no relación directa con la carrera que cursaron en la universidad. Tomando en cuenta una escala del 1 al 4 , siendo 1 equivalente a Mucho y 4 a Nada, los graduados respondieron, en un $63,9 \%$ ( $n=161)$, que su actual trabajo tiene mucha relación con los títulos que obtuvieron en la universidad; el 22,4 \% ( $n=55)$ manifestó que su trabajo algo tienen que ver en ese sentido; $7,5 \%(n=18)$, que su trabajo tiene poco que ver; y un $4,0 \%(n=10)$, que su trabajo no tiene nada que ver con lo que estudió. De estas cifras, el 11,5\% de graduados manifestaron que su trabajo tiene poco o nada de relación con su carrera universitaria.
Un resultado similar sucede con la variable Sector, en la cual, al observar las medias, se tiene que en el urbano hay una media de 1.52 , mientras que el rural es de 1.38 , indicando que en el rural existe una mayor percepción de que la carrera en la cual se tituló ha sido de mucho beneficio en su trabajo.

\section{Percepción de desempeño entre trabajo y educación.}

Al preguntarles respecto a la relación que perciben entre su desempeño en el trabajo con referencia a su educación universitaria se utilizó una escala del 1 al 5, considerando 1 como la categoría Muy en desacuerdo, y 5, Muy de acuerdo. Los graduados que ya estaban trabajando, previo a graduarse sí consideraban que sus estudios les han permitido ascender en la empresa en la que están actualmente laborando, con una media de 3.84; manifestaron que en cierta medida esto

Tabla 3. Medias de relación entre trabajo y carrera académica según sexos

\begin{tabular}{lcccc}
\hline Sexo: & N & Media & Desviación típ. & Error típ. de la media \\
\hline Femenino & 111 & 1.58 & .840 & .080 \\
Masculino & 130 & 1.42 & .769 & .067 \\
\hline
\end{tabular}

Tabla 4. Medias de relación entre trabajo y carrera académica según zona

\begin{tabular}{lcccc}
\hline Zona & $\mathbf{N}$ & Media & Desviación típ. & Error típ. de la media \\
\hline Urbano & 220 & 1.52 & .805 & .054 \\
Rural & 18 & 1.38 & .860 & .201 \\
\hline
\end{tabular}


era indiferente, pero que en parte están de acuerdo con que sus estudios les ha permito ascender. Al preguntarles si consideraban que su educación universitaria ha sido de utilidad para su actual empleo, se respondió con una media de 4.41, estando de acuerdo con este postulado; lo mismo sucedió con la pregunta ¿Considera que su formación universitaria le ha permitido desempeñarse mejor?, con una media de 4.42. Al preguntarles si consideraban que su formación les ha permitido obtener un buen empleo, se plantea una media de 3.94, indicando que, si bien les es indiferente la relación de estudio-trabajo, también están de acuerdo con que ayuda.
Alcompararestapercepciónconlasvariablessociodemográficas del estudio, se observa que, al compararla con la variable sexo, los graduados tienen una mayor percepción en comparación con las graduadas; que sus estudios les han permitido ascender dentro de su trabajo, con un rango de 94.39 de ellos en comparación con 81.27 de ellas. Un patrón similar sucede al preguntar si su formación universitaria ha sido de utilidad para su empleo, donde el lado masculino manifestó estar más de acuerdo y que considera que su formación le ha permitido tener un buen empleo.

Tabla 5. Percepción de tipos sobre estudio en posicionamiento laboral

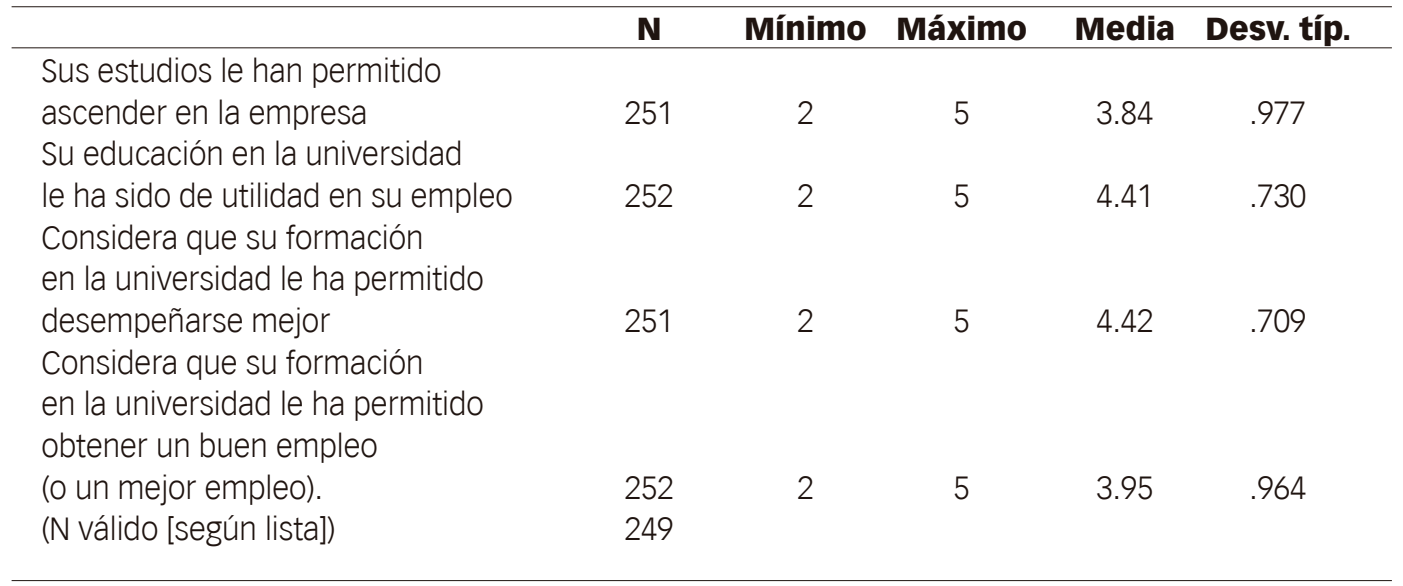

Tabla 6. Percepción de estudio en posicionamiento laboral según variables sexo

\begin{tabular}{|c|c|c|c|c|c|c|}
\hline & Sexo: & $\mathbf{N}$ & $\begin{array}{c}\text { Rango } \\
\text { promedio }\end{array}$ & $\begin{array}{c}\text { Suma de } \\
\text { rangos }\end{array}$ & $\begin{array}{c}\text { U de Mann- } \\
\text { Whitney }\end{array}$ & $\begin{array}{c}\text { Sig. asintót. } \\
\text { (bilateral) }\end{array}$ \\
\hline $\begin{array}{l}\text { Sus estudios le han } \\
\text { permitido ascender } \\
\text { en la empresa }\end{array}$ & $\begin{array}{l}\text { Femenino } \\
\text { Masculino } \\
\text { Total }\end{array}$ & $\begin{array}{c}79 \\
97 \\
176\end{array}$ & $\begin{array}{l}81.27 \\
94.39\end{array}$ & $\begin{array}{l}6420.50 \\
9155.50\end{array}$ & 3260.500 & .076 \\
\hline $\begin{array}{l}\text { Su educación en la } \\
\text { universidad le ha sido } \\
\text { de utilidad en su empleo }\end{array}$ & $\begin{array}{l}\text { Femenino } \\
\text { Masculino } \\
\text { Total }\end{array}$ & $\begin{array}{c}80 \\
97 \\
177\end{array}$ & $\begin{array}{l}85.64 \\
91.77\end{array}$ & $\begin{array}{l}6851.50 \\
8901.50\end{array}$ & 3611.500 & .378 \\
\hline $\begin{array}{l}\text { Considera que su } \\
\text { formación en la } \\
\text { universidad le ha permitido } \\
\text { desempeñarse mejor }\end{array}$ & $\begin{array}{l}\text { Femenino } \\
\text { Masculino } \\
\text { Total }\end{array}$ & $\begin{array}{c}80 \\
96 \\
176\end{array}$ & $\begin{array}{l}92.69 \\
85.01\end{array}$ & $\begin{array}{l}7415.00 \\
8161.00\end{array}$ & 3505.000 & .264 \\
\hline $\begin{array}{l}\text { Considera que su formación } \\
\text { en la universidad le ha } \\
\text { permitido obtener un buen } \\
\text { empleo (o un mejor empleo) }\end{array}$ & $\begin{array}{l}\text { Femenino } \\
\text { Masculino } \\
\text { Total }\end{array}$ & $\begin{array}{c}80 \\
97 \\
177\end{array}$ & $\begin{array}{l}87.44 \\
90.28\end{array}$ & $\begin{array}{l}6995.50 \\
8757.50\end{array}$ & 3755.500 & .700 \\
\hline
\end{tabular}


Únicamente cambia al preguntarles si su formación le ha permitido desempeñarse mejor dentro de su trabajo, donde las mujeres tuvieron una mayor percepción que este ha sido el caso, con un rango de 92.69; y 85.01 de parte del sector masculino.
Con el ítem Considera que su formación ha permitido obtener un buen empleo sucede lo mismo. Existe un mayor rango de respuesta en aquellos que manifestaron estar trabajando en contraste con los que no, que consideran que la formación no influye en la obtención de un mejor trabajo.

Tabla 7. Relación entre percepción de estudio en relación a la variable trabajo

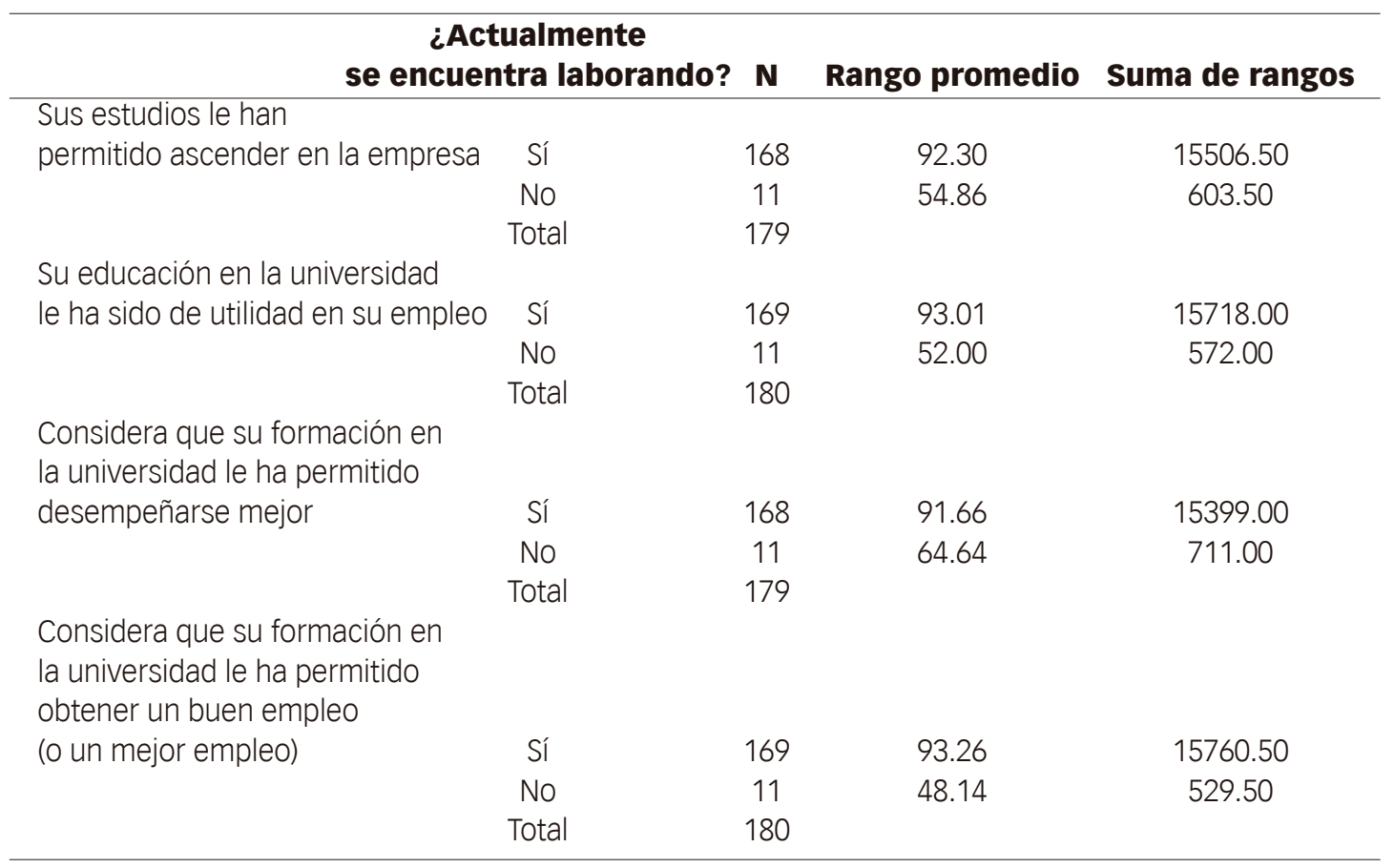

Al comparar dichas percepciones con la variable Trabajo, con una prueba de $U$ de Mann Whitney, se puede observar que existen significancias en las preguntas relacionadas con la pregunta si su estudio le ha permitido ascender en la empresa, donde hay un rango más alto entre aquellos que creen que sí les ha ayudado y que poseen trabajo, en contraste con aquellos que no tienen trabajo, donde su rango promedio es menor.

De la misma manera sucede con la pregunta si su educación ha sido de utilidad en su empleo. Las personas que manifestaron trabajan, tuvieron rangos promedios más elevados, indicando que su educación ha sido de influencia para encontrar y desempeñarse en su actual empleo.

En pocas palabras, aquellos que tienen trabajo consideran que su educación sí ha tenido influencia en la obtención y desempeño laboral, en comparación con aquellos que no poseen un trabajo, que manifiestan que la educación no influye.

\section{Conclusiones}

\section{Formación académica y empleo}

Se encontró que más de la mitad de la población de graduados encuestados (63,9\%) se encuentran laborando en un lugar relacionado con la profesión que cursó en sus estudios universitarios, mientras que un $22,4 \%$ tiene un trabajo que está relacionado de alguna manera, y un 13,7\% trabaja en un lugar que tiene poco o nada que ver con su profesión.

Son los hombres los que se están desempeñando o que ven una mayor relación entre su actual trabajo y el grado académico obtenido, en comparación a las mujeres, que tienen una menor percepción de esta relación. Un resultado similar sucede al comparar las zonas urbanas con las rurales, en el cual los graduados de la zona urbana tienen una mayor percepción de que su trabajo es afín a su especialidad, en comparación con aquellos residentes en las zonas rurales. 
Tabla 8. Prueba de significancia según la variable trabajo

\begin{tabular}{|c|c|c|c|c|}
\hline & $\begin{array}{l}\text { Sus estudios } \\
\text { le han } \\
\text { permitido } \\
\text { ascender en la } \\
\text { empresa }\end{array}$ & $\begin{array}{c}\text { Su educación } \\
\text { en la universidad } \\
\text { le ha sido } \\
\text { de utilidad } \\
\text { en su empleo }\end{array}$ & $\begin{array}{c}\text { Considera que su } \\
\text { formación } \\
\text { en la universidad } \\
\text { le ha permitido } \\
\text { desempeñarse mejor }\end{array}$ & $\begin{array}{c}\text { Considera que su } \\
\text { formación en } \\
\text { la universidad } \\
\text { le ha permitido } \\
\text { obtener un buen } \\
\text { empleo (o un } \\
\text { mejor empleo) }\end{array}$ \\
\hline \multicolumn{5}{|l|}{ U de Mann- } \\
\hline Whitney & 537.500 & 506.000 & 645.000 & 463.500 \\
\hline W de Wilcoxon & 603.500 & 572.000 & 711.000 & 529.500 \\
\hline Z & -2.426 & -2.828 & -1.879 & -2.926 \\
\hline Sig. asintót. (bilateral) & .015 & .005 & .060 & .003 \\
\hline
\end{tabular}

a. Variable de agrupación: ¿Actualmente se encuentra laborando?

\section{Percepción de la relación entre formación y crecimiento profesional}

A escala general, los graduados tienen la percepción de que existe una influencia de su formación universitaria en su desempeño y crecimiento dentro de su trabajo. Pero también parte de los graduados consideran que este es indiferente a la formación académica que obtuvo, pero sí están de acuerdo con que su formación ha sido de utilidad, funcionado como una herramienta para mejorar su desempeño en el trabajo.

En el caso de las mujeres, su formación universitaria tenía tanta relación con obtener un trabajo o incluso ascender. Incluso durante el estudio las mujeres manifestaron tener un menor salario que los hombres. Estas nociones también cambian, dependiendo de si el graduado está laborando o no. En el caso de los graduados que tienen un trabajo, dijeron que su formación no solo les ha permito obtener un buen trabajo, sino que les permitió ascender; y que además ha sido de utilidad para su desempeño laboral. Se da el caso contrario si el graduado no posee un trabajo, donde su formación no ha ayudado o permitido que logre posicionarse dentro del mercado laboral.

En este caso, la percepción de los graduados los orienta a decir que su formación universitaria les ha permitido tener las competencias y herramientas necesarias para poder desempeñarse en su trabajo, pero solo obtener su título de graduación no es garantía para obtener un buen trabajo ni para poder mejorar las condiciones laborales de su actual trabajo.

\section{Referencias}

Betnaces, L. (2007). "Nivel de posicionamiento en el mercado laboral de los egresados de la carrera de Mercadeo de la Universidad Abierta para Adultos, UAPA (Período 2004-2007)". Educación Superior. 1 y 2. Universidad Abierta Para Adultos, UAPA. Recuperado de http:// www.uapa.edu.do/docs/revista/Educ._Superior_ (enero-dic._2007).pdf

Carillo, T.; Escobar, A.; Audelo, C. (2013). "El posicionamiento laboral entre hombres y mujeres en situación de rezago educativo. El caso de la industria alimentaria de Culiacán, Sinaloa, México".

Hall, R. (1996). Organizaciones. Estructuras, procesos y resultados, 6. ${ }^{a}$ edición, Prentice Hall Hispanoamericana, S.A., México. ISBN 968-880-724-9.

Hernandez, R.; Fernández, C. y Baptista, P. (2014). Metodología de la investigación. McGraw-Hill Education. 6. ${ }^{\text {a }}$ Edición. ISBN 978-1-4562-2396-0.

Programa ALFA: América Latina Formación Académica (2006). "Manual de instrumentos y recomendaciones sobre el seguimiento de egresados. Instituto Tecnológico y de Estudios Superiores de Monterrey. México". Recuperado de http://www.mineducacion.gov. co/1621/articles-136795_pdf.pdf. ISBN: 968-891-098-8

Rentería Pérez, E. y Martínez, A. (2006 b). “ ¿Usted mismo, S.A.? $\mathrm{O}$ el posicionamiento profesional en el mercado de trabajo. Reflexiones desde el marketing y el comportamiento del consumidor". Psicología desde el Caribe, No. 18. Recuperado de http://rcientificas. 
uninorte.edu.co/index.php/psicologia/article/ view/2087 /5305. ISSN 2011-7485

Ruiz R. y Green I. (2003). “|l Curso-Taller Centroamericano sobre Metodología paraEstudios de Seguimiento de Graduados Universitarios". Memoria. II Taller. Recuperado de http:// daad.csuca.org/attachments/145_Memoria 20Final 20II
20Curso 20Egresados.doc

Universidad Modular Abierta [UMA] (2012). “Diagnóstico del posicionamiento profesional de los graduados en UMA Sonsonate como estrategia de mejora institucional. Centro Universitario de Sonsonate". 